\title{
How Do Physicians Approach Intravenous Alteplase Treatment in Patients with Acute Ischemic Stroke Who Are Eligible for Intravenous Alteplase and Endovascular Therapy? Insights from UNMASK-EVT
}

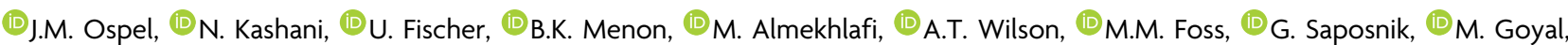
and (1) M.D. Hill

\begin{abstract}
BACKGROUND AND PURPOSE: With increasing use of endovascular therapy, physicians' attitudes toward intravenous alteplase in endovascular therapy-eligible patients may be changing. We explored current intravenous alteplase treatment practices of physicians in endovascular therapy- and alteplase-eligible patients with acute stroke using prespecified case scenarios and compared how their current local treatment practices differ compared with an assumed ideal environment.
\end{abstract}

MATERIALS AND METHODS: In an international multidisciplinary survey, 607 physicians involved in acute stroke care were randomly assigned 10 of 22 case scenarios, among them 14 with guideline-based alteplase recommendations ( 9 with level $1 A$ and 5 with level $2 \mathrm{~B}$ recommendation) and were asked how they would treat the patient: A) under their current local resources, and B) under assumed ideal conditions. Answer options were the following: 1) anticoagulation/antiplatelet therapy, 2) endovascular therapy, 3) endovascular therapy plus intravenous alteplase, and 4) intravenous alteplase. Decision rates were calculated, and multivariable regression analysis was performed to determine variables associated with the decision to abandon intravenous alteplase.

RESULTS: In cases with guideline recommendations for alteplase, physicians favored alteplase in $82.0 \%$ under current local resources and in $79.3 \%$ under assumed ideal conditions $(P<.001)$. Under assumed ideal conditions, interventional neuroradiologists would refrain from intravenous alteplase most often $(6.28 \%, \mathrm{OR}=2.40 ; 95 \% \mathrm{Cl}, 1.01-5.71)$. When physicians' current and ideal decisions differed, most would like to add endovascular therapy to intravenous alteplase in an ideal setting (196/3861 responses, 5.1\%).

CONCLUSIONS: In patients eligible for endovascular therapy and intravenous alteplase, we observed a slightly lower decision rate in favor of intravenous alteplase under assumed ideal conditions compared with the decision rate under current local resources.

ABBREVIATIONS: EVT = endovascular therapy; LVO = large-vessel occlusion

S ince 2015, endovascular therapy (EVT) and intravenous alteplase as a combined treatment have been standard of care in patients with acute ischemic stroke with large-vessel occlusion

Received September 23, 2019; accepted after revision December 11.

From the Departments of Clinical Neurosciences (J.M.O., B.K.M., M.A., A.T.W., M.M.F., M.G., M.D.H.) and Radiology (N.K., B.K.M., M.A., M.G., M.D.H.), University of Calgary, Calgary, Alberta, Canada; Division of Neuroradiology (I.M.O.), Clinic of Radiology and Nuclear Medicine, University Hospital Basel, University of Basel, Basel, Switzerland; University Hospital Bern (U.F.), Inselspital, University of Bern, Bern, Switzerland; and Division of Neurology (G.S.), Department of Medicine, St. Michael's Hospital, University of Toronto, Toronto, Ontario, Canada.

The study was funded by Stryker Inc through an unrestricted research grant to the University of Calgary. Stryker was not involved in the design, execution, analysis, and interpretation or reporting of the results.

Please address correspondence to Johanna M. Ospel, MD, Department of Clinical Neurosciences, Foothills Medical Centre, 1403 29th St NW, Calgary, AB, T2N2T9, Canada; e-mail: johannaospel@gmail.com; @johanna_ospel

Indicates article with supplemental on-line tables.

http://dx.doi.org/10.3174/ajnr.A6396
(LVO) eligible for intravenous alteplase. ${ }^{1}$ When the 5 major EVT trials were conducted, intravenous alteplase was the only approved treatment for acute ischemic stroke. ${ }^{2}$ Hence, the only acceptable trial design from an ethical standpoint was to test EVT in combination with intravenous alteplase against standard medical treatment.

A new question now arises: Does intravenous alteplase provide additional value in the setting of acute ischemic stroke due to LVO when EVT is going to be performed anyway? This question will be answered by 3 ongoing randomized controlled trials (Bridging Thrombolysis Versus Direct Mechanical Thrombectomy in Acute Ischemic Stroke [SWIFT-DIRECT], NCT03192332; Intravenous Treatment followed by Intra-Arterial Treatment versus Direct Intra-Arterial Treatment for Acute Ischaemic Stroke Caused by a Proximal Intracranial Occclusion [MR CLEAN NO IV], ISRCTN80619088; and Direct Intra-Arterial Thrombectomy in Order to Revascularize AIS Patients With Large Vessel Occlusion Efficiently in Chinese Tertiary Hospitals [DIRECT- 
MT], (NCT03469206). Current understanding suggests that there is likely modest incremental benefit only and that the benefit may mainly accrue to those who reperfuse before the planned EVT procedure. Early reperfusion in the setting of proximal LVO occurs only in relatively few patients: In the Identifying New Approaches to Optimize Thrombus Characterization for Predicting Early Recanalization and Reperfusion With IV Alteplase and Other Treatments Using Serial CT Angiography (INTERRSeCT) study, 10\% of distal ICAs and $21 \%$ of proximal M1s recanalized on follow-up CTA. ${ }^{3}$ In the Tenecteplase Versus Alteplase Before Endovascular Therapy for Ischemic Stroke (EXTEND-IA TNK) study, recanalization before EVT in proximal vessel occlusion was $10 \%$ with alteplase and $20 \%$ with tenecteplase during the time of transfer to the EVT hospital. ${ }^{4}$ The proportion may be lower if the time to EVT is fast. In the Endovascular Treatment for Small Core and Anterior Circulation Proximal Occlusion With Emphasis on Minimizing CT to Recanalization Times (ESCAPE) Trial, for instance, in which a large majority of patients were treated directly at the EVT center with very fast intravenous alteplase-to-EVT times, early recanalization was observed in only $5 \%$ of patients. ${ }^{5}$

In patients with drip-and-ship, however, when the time to EVT is longer, recanalization rates with alteplase might be higher. There is also evidence that intravenous alteplase before EVT might improve patient outcomes by reducing downstream microvascular thrombosis. ${ }^{6}$ Furthermore, complete recanalization with EVT is not guaranteed, and alteplase is most likely beneficial in cases with incomplete or failed recanalization. Current guidelines support providing both treatments concurrently when both are clinically appropriate.

However, clinical practice often does not match published evidence and guideline recommendations. How do physicians around the world currently approach intravenous alteplase treatment in the acute stroke setting in their clinical routine? This study aimed to explore real-life intravenous alteplase treatment practices of physicians across different countries and specialties using prespecified case scenarios.

\section{MATERIALS AND METHODS \\ Survey Design}

An international cross-sectional Web-based survey (UNMASKEVT) was conducted among stroke physicians to explore their current treatment practices in acute ischemic stroke. ${ }^{7}$ Participants were assigned to 10 of 22 case scenarios and asked how they would treat the patient in the given scenario: A) under their current local resources, and B) under assumed ideal conditions, with no resource restraints of any kind. In this context, current local resources could reflect monetary and infrastructural limitations (lacking access to endovascular treatment facilities), staff resources (lacking availability of technicians/nurses), and local/institutional policies (such as hospital-specific rules regarding very old patients, and so forth). Answer options were the following: 1) anticoagulation/antiplatelet therapy, 2) EVT, 3) EVT plus intravenous alteplase, and 4) intravenous alteplase. Response data were obtained from November 26, 2017, to March 27, 2018. The study was approved by the local ethics board of the University of Calgary.
Table 1: Participants' demographic baseline characteristics $(n=607)$

\begin{tabular}{lc}
\hline \multicolumn{1}{c}{ Demographics } \\
\hline Specialty (No.) (\%) \\
Neurology & $326(53.7)$ \\
Interventional neuroradiology & $173(28.5)$ \\
Neurosurgery & $81(13.3)$ \\
Others & $27(4.2)$ \\
Hospital setting (No.) (\%) & \\
Academic & $551(90.8)$ \\
Nonacademic & $56(9.2)$ \\
Age (median) (IQR) (yr) & $44(39-50)$ \\
Experience (median) (IQR) (yr) & $13(8-20)$ \\
Annual personal stroke volume (median) (IQR) & $100(50-250)$ \\
Annual personal EVT volume (median) (IQR) & $30(15-50)$ \\
Annual center tPA volume (median) (IQR) & $100(50-170)$ \\
Annual center EVT volume median (IQR) & $65(30-120)$ \\
\hline
\end{tabular}

Note:-IQR indicates interquartile range; yr, years.

\section{Survey Participants}

We invited 1330 stroke physicians (neurologists, interventional neuroradiologists, endovascular neurosurgeons, and other physicians, mostly internists and geriatricians, directly involved in acute stroke care) from 38 countries to participate in the survey. No restrictions regarding hospital setting, case volume, or experience levels were applied. Before answering the case scenarios, participants provided personal data (age, sex, years of experience in stroke treatment, estimated number of stroke patients treated per year, number of thrombectomies performed per year, geographic region, subspecialty, and hospital setting).

\section{Clinical Case Scenarios}

Twenty-two case scenarios were designed with different guideline recommendations for intravenous alteplase ( 9 with level $1 \mathrm{~A}$ and 5 with level $2 \mathrm{~B}$ recommendations, 2 without recommendations, and 6 with contraindications for intravenous alteplase) and EVT (8 with level $1 \mathrm{~A}$ and 11 with level $2 \mathrm{~B}$ recommendations, and 3 without recommendations for EVT). ${ }^{8}$ In the following analysis, only those 14 scenarios were included in which intravenous alteplase is recommended according to current American Heart Association/American Stroke Association guidelines ( 9 with level $1 \mathrm{~A}$ and 5 with level $2 \mathrm{~B}$ recommendations). Of note, the survey participants were presented with only the case vignettes; the evidence levels for EVT and alteplase treatment were not provided to them. For detailed descriptions of the case scenarios and corresponding evidence levels see On-line Table 1.

\section{Statistical Analysis}

Survey data were analyzed using descriptive statistics. Differences between categoric variables were assessed with the $\chi^{2}$ test. Multivariable logistic regression with responses clustered within the respondent was used to estimate the association between patient and physician baseline characteristics and the decision to abandon intravenous alteplase when switching from current local resources to assumed ideal conditions. Models included patient and physician baseline characteristics, namely physician age and practice experience in years; personal annual EVT and stroke treatment volume; annual center intravenous alteplase and EVT volume; geographic region; specialty and hospital setting; presentation time; baseline ASPECTS; time since symptom onset; patient age; site of occlusion; and baseline functional status. Continuous variables were converted into deciles. 
Table 2: Current and ideal decision rates in favor of intravenous alteplase overall and by evidence level ${ }^{\mathrm{a}}$

\begin{tabular}{lcccc}
\hline & EVT Level 1A & EVT Level 2B & EVT NG & Total \\
\hline tPA level 1A & & & & \\
$\quad$ Current & $85.1(1408)$ & $85.0(703)$ & - & $85.0(2111)$ \\
$\quad$ Ideal & $82.1(1359)$ & $83.0(687)$ & & $82.4(2046)$ \\
tPA level 2B & & & & \\
$\quad$ Current & $77.5(214)$ & $84.3(697)$ & $52.0(143)$ & $76.5(1054)$ \\
$\quad$ Ideal & $75.0(207)$ & $80.5(666)$ & $52.0(143)$ & $73.7(1016)$ \\
Total & & & & \\
$\quad$ Current & $84.0(1622)$ & $84.6(1400)$ & $52.0(143)$ & \\
$\quad$ Ideal & $81.1(1566)$ & $81.8(1353)$ & $52.0(143)$ & $79.3(3165)$ \\
\hline
\end{tabular}

Note:-NG indicates no guideline coverage; -, no responses in this category.

${ }^{a}$ Case scenarios with guideline-based alteplase recommendation only. Data are (\%) (No.).

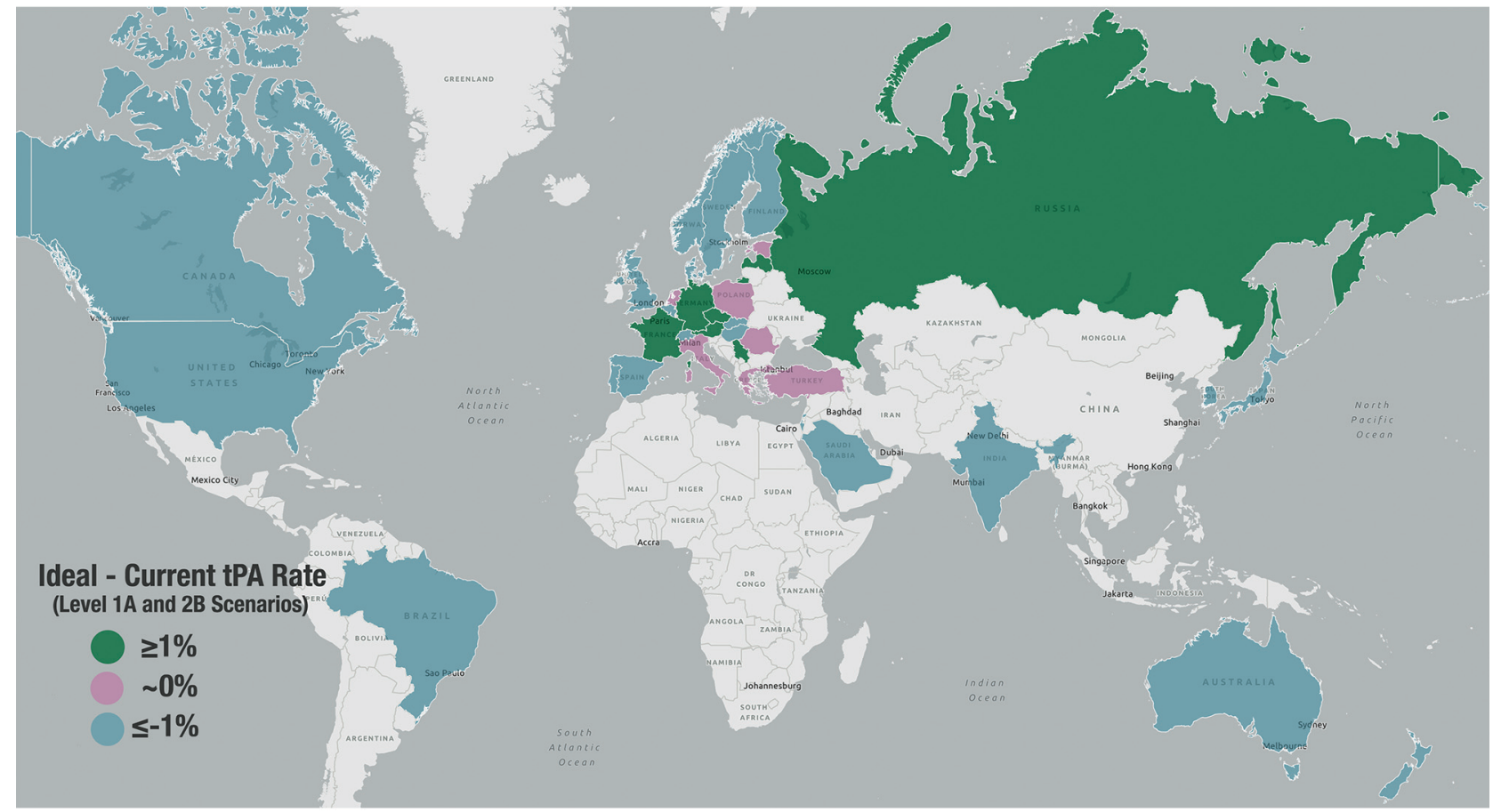

FIG 1. Resources gap (ideal minus current intravenous alteplase rates) in different countries. In green countries, the resources gap was positive $(>1 \%)$ - ie, physicians would want to treat more patients with intravenous alteplase in an ideal environment than they currently do under their current local resources. Pink countries are those with no resources gap-ie, the intravenous alteplase treatment decision under assumed ideal conditions matched the one under current local resources. In blue countries, the resources gap was negative-ie, physicians are currently treating more patients with intravenous alteplase than they would like to in an ideal environment.

All tests were 2-sided, and conventional levels of significance ( $\alpha=$ .05) were used for interpretation. Data analyses were performed in STATA 15.1 (StataCorp, College Station, Texas). Figures were created with Power BI desktop 2016 and the Mapbox Visual Plugin (Microsoft, Redmond, Washington).

\section{RESULTS}

\section{Response Rate and Participants' Demographic Characteristics}

A total of 607 physicians of different subspecialties from 38 countries completed the survey (response rate, $45.6 \%$ ). Table 1 provides an overview about participants' demographic baseline characteristics. A total of 6070 responses were obtained for the 22 case scenarios, among them 3861 for the 14 scenarios with the guideline-based alteplase recommendation. Only the latter ones were included in the following analysis. The number of responses, evidence level, and alteplase decision rates for each single case scenario can be found in the On-line Table 2.

\section{Current and Ideal Decision Rates in Favor of Intravenous Alteplase}

Table 2 shows the overall and evidence level-specific intravenous alteplase decision rates under current local resources and assumed ideal conditions. Overall, slightly fewer physicians would treat with intravenous alteplase under assumed ideal conditions $(79.3 \%)$ compared with their decision under current local resources (82.0\%; risk difference, $2.7 \%$; $P<.001$; Table 2). This decision held true for neurologists, who constituted the largest group among all specialties, as well (85.7 versus $87.7 \%, P<.001$ ). Only in case scenarios with level $2 \mathrm{~B}$ evidence for intravenous alteplase and without guideline-based EVT recommendations would 


\section{Current Local Resources}

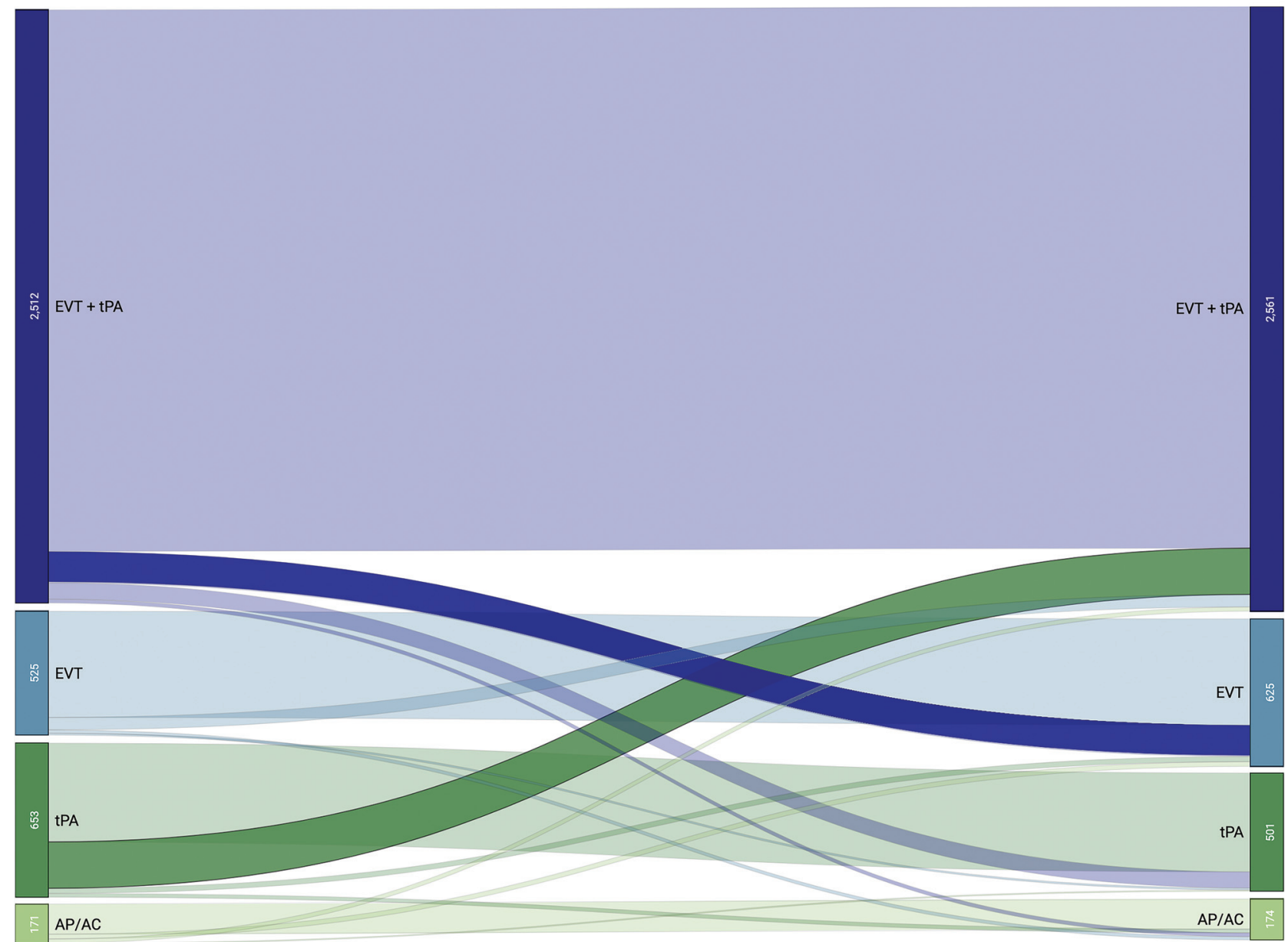

FIG 2. Changes in decision-making from current local resources (left) to assumed ideal conditions (right). The width of the streams is proportional to the number of changes. tPA, intravenous alteplase; AP/AC, antiplatelet or anticoagulation therapy.

participants proceed equally often with EVT under assumed ideal conditions and current local resources (52.0\%, Table 2). Fig 1 illustrates the resources gap (ideal minus current intravenous alteplase decision rates) by country. In most countries, current intravenous alteplase decision rates are higher than the ideal rates.

\section{Changes in Treatment Decision from Current Local Resources to Assumed Ideal Conditions}

Fig 2 illustrates how physicians' decision-making changed between current local resources and assumed ideal conditions. Overall, patients in most case scenarios would have received the same treatment under current local resources and assumed ideal conditions. Among those physicians who changed their treatment approach under assumed ideal conditions, most of them would have liked to add EVT to intravenous alteplase (dark green stream in Fig 2; 196/3861 responses, 5.1\%). The second-largest change occurred from alteplase plus EVT to EVT alone (dark blue stream in Fig 2; 133/3861 responses, $3.4 \%$ ). Overall, alteplase would have been added in $84 / 3861$ (2.2\%) scenarios under assumed ideal conditions, while it would have been removed in 187/3861 (4.8\%) scenarios.

\section{Physician and Patient Characteristics Associated with the Decision to Abandon Intravenous Alteplase under Assumed Ideal Conditions}

Multivariable logistic regression analysis revealed that of all physician characteristics, only the specialty of interventional neuroradiology was significantly associated with the decision to abandon intravenous alteplase (OR $=2.40$; $95 \% \mathrm{CI}, 1.01$ 5.71; reference category neurology) when switching from current local resources to assumed ideal conditions. Fig 3 shows the percentage of physicians who would drop and add intravenous alteplase when switching from current local resources to assumed ideal conditions. In an ideal environment, all specialties would drop intravenous alteplase more often than they would add it, and this choice was most pronounced in interventional neuroradiologists and neurosurgeons. Other physician factors (age, practice experience in years, personal annual EVT and stroke treatment volume, annual center intravenous alteplase and EVT volume, geographic region, hospital setting) and patient factors (presentation time, baseline ASPECTS, time since symptom onset, patient age, site of occlusion, baseline functional status) were not significantly associated with the decision to abandon intravenous alteplase. 

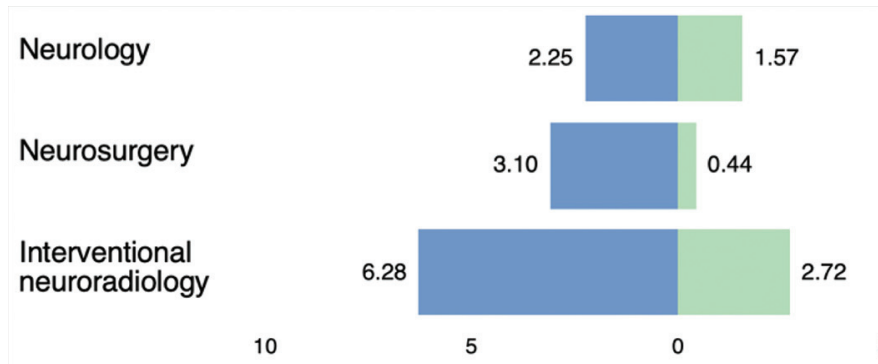

FIG 3. Percentage of physicians who would drop intravenous alteplase (blue bars) and add intravenous alteplase (green bars) by specialty.

\section{DISCUSSION}

In this multidisciplinary survey, we evaluated how physicians around the world approach intravenous alteplase treatment in patients eligible for EVT and alteplase treatment and how they would like to change their treatment practice when assuming ideal conditions (ie, in the absence of any external limitations). We found slightly lower reported intravenous alteplase decision rates under assumed ideal conditions compared with decision rates under current local resources (2.7\% risk difference, Table 2). This finding mostly held true within individual countries as well (Fig 1). We suspect that under ideal conditions, EVT would be more readily available and that this reduction in reported alteplase treatment rates shows physicians' demands for a single method of reperfusion that has a high success rate. This behavior may reflect binary thinking: One solution is preferred when, in fact, the 2 treatments may be synergistic. While intravenous alteplase has been proven to be an effective treatment in acute ischemic stroke, and although recanalization rates for LVO are lower than for distal occlusions (5-10\%), ${ }^{9,10}$ concurrent administration of alteplase and the use of EVT afford the possibility of early recanalization, even in patients with LVO. ${ }^{10}$ Furthermore, medical thrombolysis may facilitate $\mathrm{EVT}^{11}$ through a reduction in a thrombus-endothelial interaction, leading to more effective firstpass reperfusion and by causing thrombolysis of distal emboli (whether such emboli are iatrogenic or due to the original stroke event). ${ }^{6,12}$ Indeed, our results show that among those physicians who changed their treatment decision when switching from current local resources to assumed ideal conditions, most of them would opt for a combined approach and add EVT to intravenous alteplase (Fig 2). This increase in EVT rates probably occurred because access to EVT is currently limited in many hospital settings.

In contrast, there are potential negative attributes of treatment with alteplase. The efficacy of both EVT and medical thrombolysis with alteplase is highly time-dependent, and difficulties in determining intravenous alteplase eligibility and initial transfer to a primary stroke center for its administration can substantially delay EVT, thereby reducing EVT efficacy and ultimately worsening patient outcome. ${ }^{13-16}$ Moreover, the absolute rate of major hemorrhage is slightly higher when alteplase is used, though it is not statistically significant. ${ }^{17,18}$ Both EVT and intravenous alteplase may cause thrombus fragmentation and distal embolization, but when alteplase is used primarily, it may convert a relatively
5

straightforward M1 embolectomy into a more difficult procedure, involving $\geq 2$ middle cerebral artery branch occlusions. This feature potentially increases treatment costs without improving outcomes. ${ }^{19}$ These considerations could potentially explain why ideal alteplase rates were significantly lower (both in the overall group of participants as well as among neurologists, who constituted the largest specialty subgroup) than ideal rates, despite formal evidence for alteplase treatment. The difference was statistically significant but small and of unclear clinical significance. However, depending on the results of MR CLEAN NO IV, DIRECT-MT, and SWIFT-DIRECT, this gap might grow further in the future. The specialty of interventional neuroradiology reported a significantly increased probability of dropping intravenous alteplase when switching from current local resources to assumed ideal conditions. This is likely due to treatment familiarity, but it could also potentially reflect a bias of binary thinking in treatment decision-making. The differences between current and ideal intravenous alteplase treatment rates in different countries, and particularly between North America and Europe (Fig 1), are most likely multifactorial and related to infrastructure and local policies. While the North American and European guidelines mostly agree in their alteplase and EVT treatment recommendations, local/institutional policies might differ across countries. Because neurologists were the largest group in almost all countries, physician specialty is probably not the main reason for these discrepancies. The exact reasons for the observed differences can, however, not be determined in this study and should be made subject to further research. Particularly in developing countries, many of which were not captured in this survey, access to EVT is still lacking and intravenous alteplase remains the mainstay of acute stroke therapy.

Our study has several limitations that deserve comment. The overall response rate of this survey was modest at $45.6 \%$. Although the difference between current and ideal intravenous alteplase decision rates was statistically significant, the overall difference was small and the clinical significance of these discrepancies remains to be clarified. Enrollment was based on institutional networks and cooperations, potentially leading to a selection bias. Neurosurgeons and interventional neuroradiologists, who made up $42 \%$ of the participants, do not decide about alteplase treatment in most practice settings. We tried to design case scenarios that reflect clinical routine as realistically as possible, but a case-based survey cannot fully capture real-life decision-making. We did not obtain any information about the specific nature of external limitations that cause the resource gaps observed in our study. Furthermore, it is possible that lack of access to EVT in participants' current local setting, as is the case in many countries that were represented in this survey, also influences their treatment decisions under assumed ideal conditions: Physicians who do not have access to EVT might not be familiar with this treatment and might, thus, not feel comfortable offering it even if 
they could. Last, the results of this survey are a snapshot in time, whereas clinical decision-making is a dynamic construct; it is possible, for instance, that more liberal EVT treatment practices in the future will cause further reactive changes in intravenous alteplase treatment practice. Despite its limitations, this study helps to explore how intravenous alteplase treatment in patients with acute ischemic stroke is currently approached across a broad international, multidisciplinary spectrum and provides a helpful context for the 3 ongoing randomized trials.

\section{CONCLUSIONS}

Participants of this survey stated that they would treat slightly more patients with intravenous alteplase in their current local setting than they would in an ideal environment. These differences, though statistically significant, were, however, small, and their clinical significance remains unclear at the moment. Physicians who changed their decisions under assumed ideal conditions mostly wanted to add EVT in addition to intravenous alteplase.

\section{ACKNOWLEDGMENTS}

The authors are most grateful to all physicians participating in the study.

Disclosures: Johanna M. Ospel—RELATED: Grant: Julia Bangerer-Rhyner Foundation, University of Basel Forschungsfonds, Freiwillige Akademische Gesellschaft Basel, Comments: research scholarship. Nima Kashani-UNRELATED: Employment: Calgary University. Urs Fischer-UNRELATED: Consultancy: Medtronic, Stryker, CSL Behring*; Grants/Grants Pending: Medtronic, Comments: SWIFT DIRECT trial.* Bijoy K. Menon-OTHER RELATIONSHIPS: I hold a patent on systems of triage in acute stroke and stock in Circle Neuovascular Imaging. Mona M. Foss-RELATED: Consulting Fee or Honorarium: University of Calgary, Comments: honorarium for statistical consulting. Gustavo Saposnik-RELATED: Support for Travel to Meetings for the Study or Other Purposes: University of Calgary; UNRELATED: Consultancy: Roche, Servier, Celgene; Employment: St. Michael's Hospital; Grants/Grants Pending: Roche, Servier; Payment for Lectures Including Service on Speakers Bureaus: Celgene, Servier; Travel/Accommodations/Meeting Expenses Unrelated to Activities Listed: Roche, Comments: to attend ECTRIMS 2019. Mayank GoyalRELATED: Grant: Stryker, Comments: unrestricted research grant for conduction of UNMASK EVT*; UNRELATED: Consultancy: Medtronic, Stryker, Mentice, MicroVention, Comments: consulting regarding acute stroke intervention and products; Patents (Planned, Pending or Issued): GE Healthcare, Comments: licensing agreement for systems of acute stroke diagnosis. Michael D. Hill—RELATED: Grant: Stryker, Comments: grant to the University of Calgary for the UNMASK-EVT project*; UNRELATED: Board Membership: Coalition for National Science Funding Canadian Neuroscience Federation (CNSF) and Canadian Stroke Consortium (CSC), Comments: not-for-profit boards in stroke and neurology; Consultancy: Merck, Comments: paid consultancy for adjudicating clinical trial outcome events; Grants/ Grants Pending: multiple sources, Comments: multiple grants from both industry and public sources for clinical trials*; Patents (Planned, Pending or Issued): patent on stroke care, Comments: patent granted. No money paid; Stock/Stock Options: Calgary Scientific Inc (CSI), Comments: stock owner in CSI, imaging and computerrendering company. Mohammed Almekhlafi—RELATED: Grant: Stryker. *Money paid to the institution.

\section{REFERENCES}

1. Goyal M, Menon BK, van Zwam WH, et al; HERMES Collaborators. Endovascular thrombectomy after large-vessel ischaemic stroke: a meta-analysis of individual patient data from five randomised trials. Lancet 2016;387:1723-31 CrossRef Medline

2. Campbell BC, Meretoja A, Donnan GA, et al. Twenty-year history of the evolution of stroke thrombolysis with intravenous alteplase to reduce long-term disability. Stroke 2015;46:2341-46 CrossRef Medline

3. Menon BK, Al-Ajlan FS, Najm M, et al; INTERRSeCT Study Investigators. Association of clinical, imaging, and thrombus characteristics with recanalization of visible intracranial occlusion in patients with acute ischemic stroke. JAMA 2018;320:1017-26 CrossRef Medline

4. Campbell BC, Mitchell PJ, Churilov L, et al; EXTEND-IA TNK Investigators. Tenecteplase versus alteplase before thrombectomy for ischemic stroke. N Engl J Med 2018;378:1573-82 CrossRef Medline

5. Goyal M, Demchuk AM, Menon BK, et al; ESCAPE Trial Investigators. Randomized assessment of rapid endovascular treatment of ischemic stroke. $N$ Engl J Med 2015;372:1019-30 CrossRef Medline

6. Desilles JP, Loyau S, Syvannarath V, et al. Alteplase reduces downstream microvascular thrombosis and improves the benefit of large artery recanalization in stroke. Stroke 2015;46:3241-48 CrossRef Medline

7. Saposnik GM, Kashani N, Wilson AT, et al. Factors associated with the decision-making on endovascular thrombectomy for the management of acute ischemic stroke. Stroke 2019;50:2441-47 CrossRef Medline

8. Powers WJ, Rabinstein AA, Ackerson T, et al; American Heart Association Stroke Council. 2018 Guidelines for the Early Management of Patients with Acute Ischemic Stroke: A Guideline for Healthcare Professionals from the American Heart Association/American Stroke Association. Stroke 2018;49:e46-110 CrossRef Medline

9. Mueller L, Pult F, Meisterernst J, et al. Impact of intravenous thrombolysis on recanalization rates in patients with stroke treated with bridging therapy. Eur J Neurol 2017;24:1016-21 CrossRef Medline

10. Seners $P$, Turc G, Maier B, et al. Incidence and predictors of early recanalization after intravenous thrombolysis: a systematic review and meta-analysis. Stroke 2016;47:2409-12 CrossRef Medline

11. Guedin P, Larcher A, Decroix JP, et al. Prior IV thrombolysis facilitates mechanical thrombectomy in acute ischemic stroke. J Stroke Cerebrovasc Dis 2015;24:952-57 CrossRef Medline

12. Davalos A, Pereira VM, Chapot R, et al; Solitaire Group. Retrospective multicenter study of Solitaire FR for revascularization in the treatment of acute ischemic stroke. Stroke 2012;43:2699-705 CrossRef Medline

13. Choi PM, Desai JA, Kashyap D, et al. Are all stroke patients eligible for fast alteplase treatment? An analysis of unavoidable delays. Acad Emerg Med 2016;23:393-99 CrossRef Medline

14. Dhaliwal J, Ferrigno B, Abiola O, et al. Hospital-based intervention to reduce tPA administration time. Interdisciplinary Neurosurgery 2019;15:15-18. CrossRef

15. Kamal N, Sheng S, Xian Y, et al. Delays in door-to-needle times and their impact on treatment time and outcomes in Get with the Guidelines-Stroke. Stroke 2017;48:946-54 CrossRef Medline

16. Mohamad NF, Hastrup S, Rasmussen M, et al. Bypassing primary stroke centre reduces delay and improves outcomes for patients with large vessel occlusion. Eur Stroke J 2016;1:85-92 CrossRef Medline

17. Wardlaw JM, Murray V, Berge E, et al. Thrombolysis for acute ischaemic stroke. Cochrane Database Syst Rev 2014;7:CD000213 CrossRef

18. Yaghi S, Eisenberger A, Willey JZ. Symptomatic intracerebral hemorrhage in acute ischemic stroke after thrombolysis with intravenous recombinant tissue plasminogen activator: a review of natural history and treatment. JAMA Neurol 2014;71:1181-85 CrossRef Medline

19. Rai AT, Boo S, Buseman C, et al. Intravenous thrombolysis before endovascular therapy for large vessel strokes can lead to significantly higher hospital costs without improving outcomes. $J$ Neurointerv Surg 2018;10:17-21 CrossRef Medline 